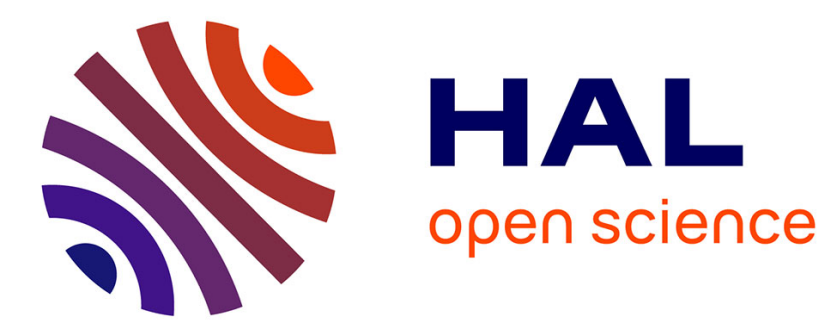

\title{
Concurrence générationnelle et prix immobiliers
}

Arnaud Simon, Yasmine Essafi

\section{To cite this version:}

Arnaud Simon, Yasmine Essafi. Concurrence générationnelle et prix immobiliers. 2015. halshs$01138074 \mathrm{v} 3$

\section{HAL Id: halshs-01138074 \\ https://shs.hal.science/halshs-01138074v3}

Preprint submitted on 25 Mar 2016

HAL is a multi-disciplinary open access archive for the deposit and dissemination of scientific research documents, whether they are published or not. The documents may come from teaching and research institutions in France or abroad, or from public or private research centers.
L'archive ouverte pluridisciplinaire HAL, est destinée au dépôt et à la diffusion de documents scientifiques de niveau recherche, publiés ou non, émanant des établissements d'enseignement et de recherche français ou étrangers, des laboratoires publics ou privés. 


\title{
Concurrence générationnelle et prix immobiliers
}

\author{
Arnaud Simon, Université Paris Dauphine (auteur de contact) \\ Arnaud.simon@dauphine.fr \\ Yasmine Essafi, BNP Paribas Real Estate \\ yasmine.essafi@bnpparibas.com
}

\section{Résumé}

La coïncidence entre 1997 et 2007 d'un grand nombre de futurs retraités avec la très forte hausse des prix du résidentiel est-elle fortuite ? En se basant sur des éléments temporels et géographiques, complétés par un modèle de panel, cet article tend à confirmer l'existence d'une causalité démographique. La prise en compte de ces facteurs démographiques, en absolu et en structure, fait de plus apparaitre comme minimes les effets des taux, des revenus, de la construction et du divorce, sur les prix. Dans le contexte actuel de 'papy-boom', le modèle suggère une lente érosion des prix. Toutefois, une question se pose dès aujourd'hui, celle d'une concurrence intergénérationnelle. Alors que près de $75 \%$ des retraités sont propriétaires, l'orientation pro 'papy-boomers' de la politique du logement mérite d'être interrogée. Le parc résidentiel valant 6300 milliards d'euros et ayant enregistré une plus-value de 3700 milliards, on ne saurait le considérer comme un horsbilan. Crise, déflation, austérité et 'papy-boom' ne seraient-ils pas des synonymes?

\begin{abstract}
$\underline{\text { Abstract }}$
Is the simultaneity, between 1997 and 2007, of a great number of future retired people and the important rise of residential prices, a random effect? In this article, temporal and geographical elements, completed with a panel model, allow answering negatively; a causal relation exists. Taking into account demographic factors such as size and structure of the population leads to a minimization of rates, wages and construction roles in determining residential prices. In the present 'grandpa-boom' environment, the model indicates that this factor could generate a slow price decrease. However, the question of intergenerational competition arises. While $75 \%$ of the retired people are owners, the pro 'grandpa-boomers' orientation of the fiscal and housing policies has to be questioned. The residential building stock can be estimated around 6300 and it registered a value creation of 3700 billion euros; it cannot be considered as being off balance. Would not deflation, austerity and 'grandpa-boom' be synonyms?
\end{abstract}

Mots-clés : prix immobiliers, démographie, vieillissement, inégalité intergénérationnelle

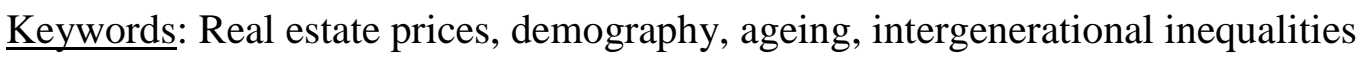

JEL: J11, R21, R23 


\section{Introduction}

Le prix des logements, c'est-à-dire la possibilité de devenir propriétaire d'une manière raisonnable sans avoir à consentir des efforts démesurés, est un enjeu majeur de la cohésion sociale. Or depuis 1998 les prix ont augmenté de près de $250 \%$ en France. Cette hausse a également été observée dans de nombreux autres pays : Italie, Royaume-Uni, Pays-Bas, Danemark, Australie, Canada, EtatsUnis... Elle n'a cependant pas été systématique et des pays comme le Japon et l'Allemagne ont présenté des mouvements baissiers significatifs.

Des réflexions développées dans l'économie du travail permettent de mettre en perspective cette question. On considère assez souvent que les 'insiders' du marché du travail, c'est-à-dire ceux qui ont un travail relativement stable, et les 'outsiders', personnes en situation de précarité ou au chômage, ont des intérêts divergents voire opposés. Cela engendre assez fréquemment un renforcement des barrières à l'entrée, c'est-à-dire une difficulté accrue à l'obtention d'un CDI. Or aujourd'hui devenir propriétaire impose de franchir une barrière à l'entrée majeure, celle du prix. Peut-on trouver une logique à ce phénomène ?

L'analyse du prix des biens résidentiels fait intervenir trois dimensions : économique, spatiale et démographique. La première dimension mobilise des explications relatives à la croissance économique, aux taux d'intérêts, à la plus ou moins grande facilité d'octroi des crédits, au niveau de la construction ainsi que des éléments fiscaux. La seconde s'interroge sur la manière dont se déclinent sur le territoire les différentes dynamiques : zones de concentration de la richesse, zones d'éviction liées à des phénomènes de paupérisation et/ou de désertification. Elle relève des politiques d'aménagement du territoire.

Par ce travail, nous souhaitons attirer l'attention sur la troisième dimension, c'est-à-dire le facteur démographique, qui nous semble être central. Quel est l'impact des changements démographiques sur le marché immobilier résidentiel français, et plus particulièrement quels sont les effets de distorsion dus aux classes d'âge plus ou moins nombreuses ? Car le baby-boom est aujourd'hui devenu un papy-boom. En d'autres termes, peut-on mettre en rapport le déplacement de la cohorte des baby-boomers avec la plus ou moins grande facilité à devenir propriétaire ? Ne seraient-ils pas aujourd'hui devenus les 'insiders' de la propriété immobilière?

Pour tenter de répondre à cette question nous présentons dans la section 2 le cadre conceptuel dans lequel s'inscrivent ces réflexions. Les trois dimensions : économique, spatiale et démographique, qui a notre sens sont constitutives du prix immobilier, y sont détaillées avec leurs références bibliographiques. Les réflexions théoriques relatives à l'économie du cycle y sont centrales. La section suivante met l'accent sur le facteur démographique en discutant les caractéristiques des cohortes du papy-boom. Elle fournit les premiers éléments chiffrés relatifs à un lien causal entre le déplacement temporel de ces cohortes et la dynamique du prix des logements : coïncidence des retournements de tendances, intuition d'un lien sur la période [1975;2010], déclinaison spatiale du phénomène. Nous précisons ensuite empiriquement ce lien en ayant recours à une approche en données de panel, de niveau départemental, sur la période [2000-2013]. L'enseignement principal réside dans le fait que ce sont surtout les variables démographiques qui sont explicatives du prix, et en particulier le ratio structurel OLDDEP qui rapporte l'effectif des personnes de plus de 60 ans, à celui des personnes entre 20 et 60 ans. La section 5 explore les déclinaisons spatiales de ce phénomène sur des sous-groupes de départements : urbains, ruraux, jeunes, âgés et par niveau de prix. La partie 6 fera la synthèse et proposera une vue d'ensemble de la situation. L'impact des 
cohortes du baby-boom sur les prix immobiliers y sera récapitulé et mis en rapport avec le thème de la concurrence générationnelle. Les politiques du logement y seront interrogées quant à leur orientation déséquilibrée et pro papy-boomers. La section 7 conclura, en particulier sur l'idée que la distribution intergénérationnelle de la propriété ne saurait rester un impensé, un hors-bilan.

\section{Prix immobiliers : une perspective démographique, économique et spatiale}

Cette section inscrit la problématique dans l'économie du cycle de vie et introduit les trois axes d'analyse. La Figure 1 synthétise ces éléments et résume le modèle conceptuel.

a. Economie du cycle de vie, principes généraux, 'melt-down' et 'melt-up'

Le comportement économique d'un individu (épargne, consommation, investissement) dépend de de ses besoins personnels, de ses besoins professionnels et de ses contraintes budgétaires. Or durant les différentes phases de sa vie ces éléments vont varier. La théorie du cycle de vie (ANDO, MODIGLIANi (1963)) pose comme principe central que les individus auront tendance à lisser leur consommation entre ces différentes phases. Si au début de la vie active le niveau de revenu d'un individu est faible, il augmente cependant peu à peu. Il va alors tenter, à l'aide de l'endettement, de se constituer un patrimoine en achetant des actifs financiers et/ou immobiliers. Une fois à la retraite, ses revenus se réduisant, il utilisera le patrimoine qu'il s'est constitué pour soutenir son niveau de consommation.

Au niveau agrégé, l'ensemble de ces décisions individuelles influe sur l'offre et la demande globale des actifs, et par conséquent sur leur prix. En particulier, la taille et la structure de la population vont exercer des pressions sur les prix (ALLAIS (1947), SAMUELSON (1958), DiAmOND (1965)). Le terme 'melt-up' désigne la montée du prix des actifs produit par une population active nombreuse, le terme 'melt-down' désigne le mouvement de baisse lorsque la population vieillit. Les fonds de pension sont une illustration de ce mécanisme; ils peuvent être en période de collecte nette (beaucoup d'épargnants, peu de pensions à verser) ou bien en période de décollecte (situation inversée). Dans le premier cas ils sont structurellement acheteurs de titres et devraient pousser les prix à la hausse, dans le second structurellement vendeurs et donc exercer une pression à la baisse.

La question de la manifestation d'un 'melt-down' sur les marchés de capitaux est un champ de recherche actif. ABEL (2003) a développé un modèle théorique visant à étudier l'impact des babyboomers sur ces marchés; il affirme que les prix des actions devraient baisser avec le papy-boom. JAMEL et QUAYES (2004) ont aussi montré que la structure démographique, et plus précisément, la taille de la population active, avaient une influence directe sur les prix des actions américaines et anglaises. Cet impact est aussi confirmé par CAMPBELL (2007) qui trouve des liens clairs entre l'âge moyen de la population américaine et le rendement de long-terme de l'indice S\&P500. ANG et MADDALONI (2005) et POTERBA $(2001,2004)$ trouvent également des relations significatives mais ils indiquent que cela n'est pas systématique et qu'il peut exister des variations entre pays. Par contre MAREKWICA et al. (2011), en s'appuyant sur un modèle macroéconomique multifactoriel, ne trouvent pas de relations robustes entre chocs démographiques et rendements 
financiers. Il convient aussi de rappeler que les particuliers ne sont pas les seuls acteurs sur les marchés financiers ; la question se poserait donc plutôt en termes d'un facteur qui contribuerait à la variation des cours, un facteur parmi d'autres.

\section{b. Cycle de vie et logement}

Le logement trouve à s'inscrire aisément dans cette théorie. Si les parcours résidentiels peuvent être multiples et différenciés, l'objectif que beaucoup d'individus tentent de réaliser consiste cependant à devenir propriétaire de leur résidence principale, afin de ne plus avoir de mensualités bancaires à rembourser au moment de leur retraite. La réduction de leurs revenus serait alors contrebalancée par la fin du paiement de la mensualité du crédit.

A une échelle internationale, l'étude de TAKATS (2012) a permis de tester la pertinence du 'meltup' et du 'melt-down' pour le logement. Un modèle en données de panel sur la période [1970 ; 2009], pour 22 pays avancés économiquement ${ }^{1}$, a été estimé. Ce modèle indique que les éléments démographiques ont été à l'origine d'une augmentation moyenne des prix des logements dans ces pays de $+0,3 \%$ par an, sur ces quarante dernières années, et que ces mêmes facteurs devraient produire une baisse de $-0,8 \%$ par an pour les quarante prochaines années.

Dans cet article, un modèle à générations imbriquées est mentionné. Une relation importante mérite d'être relevée :

$$
(1+r)=(1+g) *(1+d)
$$

Cette formule indique que, dans la perspective de l'économie du cycle de vie, le prix de l'actif immobilier s'accroit à un taux $\mathrm{r}$ qui est fonction de deux facteurs : un facteur économique g, et un facteur démographique d. Il arrive assez souvent que les études et les recherches sur le logement se concentrent sur le facteur g (PIKETTY (2013), BonNET ET AL. (2014)). Or dans le contexte de papy-boom que nous connaissons aujourd'hui on ne saurait oublier le facteur $\mathrm{d}$. Comme nous le verrons dans la suite de cet article, il se révélera même être le facteur principal dans l'explication de l'évolution du prix des logements pour le cas de la France. Cette relation fondamentale va nous permettre d'organiser le choix des variables explicatives.

\footnotetext{
${ }^{1}$ Allemagne, Australie, Autriche, Belgique, Canada, Corée du Sud, Danemark, Espagne, États-Unis, Finlande, France, Grèce, Irlande, Italie, Japon, Nouvelle-Zélande, Norvège, Pays-Bas, Portugal, Royaume-Uni, Suède, Suisse.
} 
Figure 1 : Modèle conceptuel pour l'étude du prix des logements

Cette figure synthétise le modèle conceptuel développé dans cet article. Les trois sphères d'influence, économique (g), démographique (d) et spatiale y sont représentées avec les variables opérationnelles retenues et les problématiques associées. Pour les variables, les signes attendus sont également indiqués.

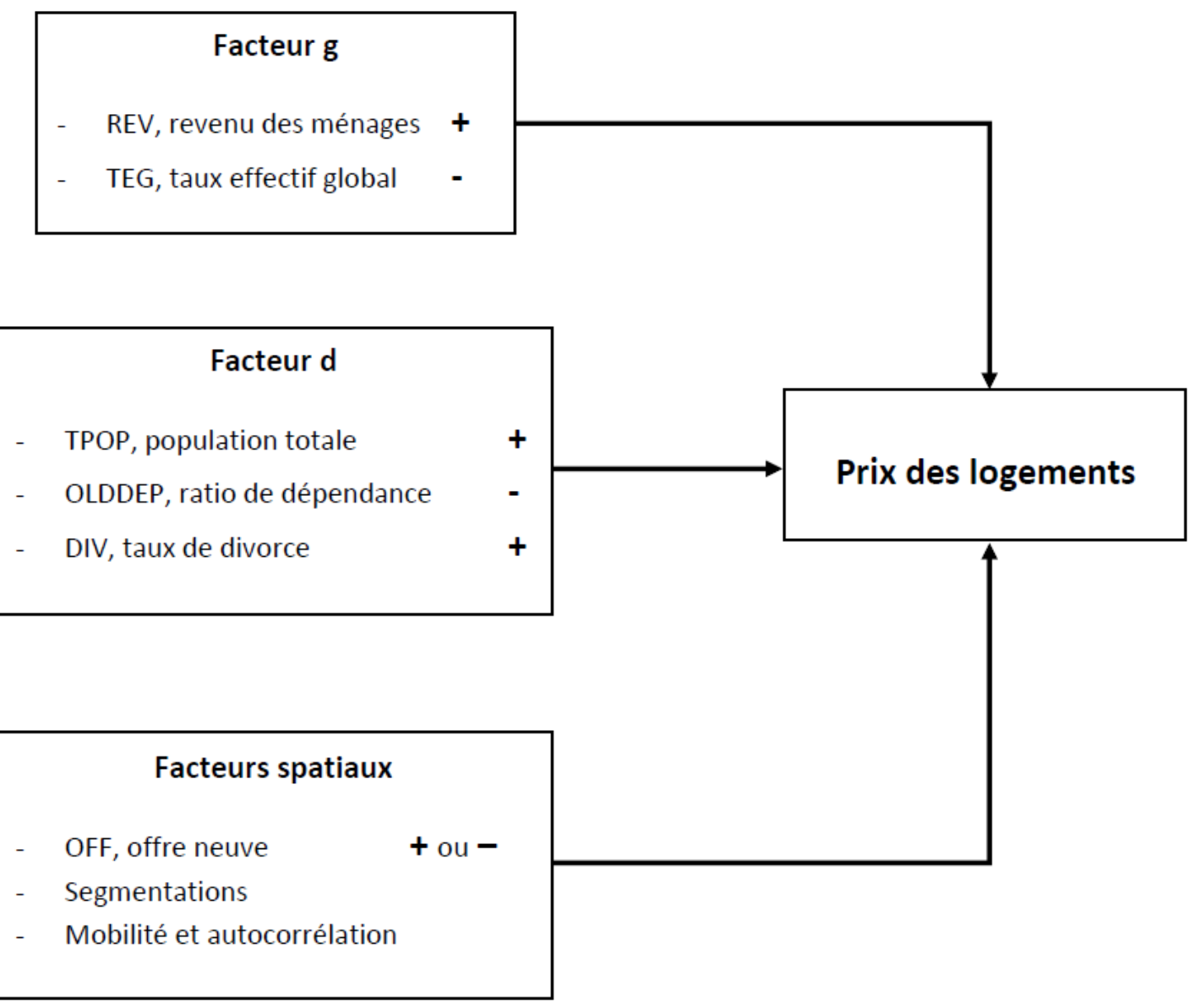

c. Le facteur d et le choix des variables démographiques

La prise en compte très partielle du facteur $d$ dans les recherches sur le logement en France ces vingt dernières années impose d'approfondir un peu la revue de littérature afférente à cet élément. Deux périodes de recherche peuvent être dégagées.

Premier temps de recherche : 1989-1996

MANKIW et WeIL (1989) peuvent être considérés comme un point de départ. Ces auteurs ont étudié l'impact du facteur démographique sur le marché immobilier américain. Ils ont modélisé la demande de logement comme une fonction de l'âge, et plus spécifiquement comme une fonction de la cohorte de la population active. Ils indiquent qu'une demande plus forte, provenant d'une cohorte plus importante produit une hausse des prix, et que par contre lorsque cette classe d'âge 
arrive à la retraite une baisse des prix se manifeste. Ces résultats ont été interrogés par HENDERSHOTT (1991) qui a critiqué la spécification du modèle : il n'incluait en effet pas suffisamment de variables, et en particulier pas de variables rendant compte de l'évolution de la richesse de la population ${ }^{2}$. HoLland (1991) indiqua pour sa part que le lien détecté entre les variables démographiques et la demande de logement pourrait être artificiel, car obtenu avec des variables non-stationnaires. Il indiqua également qu'il convient de considérer la réponse de l'offre. Cela a conduit DI PASQUALE et WHEATON (1994) à intégrer cet élément dans leur modèle structurel offre-demande.

Il semble que l'effet démographique puisse présenter une variabilité suivant le pays considéré. ENGELHARDT et POTERBA (1991), reproduisant l'approche initiale de MANKIW et WeIL (1989), n'ont ainsi pas trouvé de relation significative entre l'indice démographique pour la demande de logement et les prix immobiliers au Canada, alors que ce pays présente une structure similaire à celle des Etats-Unis. Quant à OHTAKE et SHINTANi (1996), qui ont aussi reproduit le modèle de base en utilisant des données japonaises, ils indiquent que l'influence démographique serait limitée dans le temps et qu'elle serait contrebalancée dès que l'offre augmenterait en réponse à la variation de la demande. Ils considèrent que le facteur démographique a davantage un impact sur la dimension du parc immobilier que sur le prix du mètre carré.

Les recherches et la critique produites au cours de ce premier temps ont permis d'arriver à une certaine maturité dans la manière de spécifier le modèle. Les résultats obtenus semblent valider l'existence du lien entre démographie et prix immobiliers, même si des réserves sont énoncées.

\section{Deuxième temps de recherche : Ré-interrogation contemporaine de la question}

Les articles récents aboutissent à des résultats plus nets. NAKAMURA et SAITA (2007) sont parvenus à montrer que les changements démographiques impactaient effectivement bien les prix immobiliers japonais, et cela même dans le long-terme. En utilisant des données autrichiennes et en ajustant le modèle de MANKIW et WEIL (1989), LEE et al. (2001) ont eux aussi trouvé des éléments de preuve. Dernièrement, NiSHIMURA (2011) ainsi que SAITA et al. (2013) sur données japonaises, et TAKATS (2012) avec une étude sur 21 pays et sur 40 ans, ont confirmé ces éléments. D'autres auteurs tels ERMisch (1996), ForTin et LECLERC (2002), SHIMIZU et WATANABE (2010), NEUTEBOOM et BROUNEN (2007) concluent aussi positivement.

Il n'est pas sans intérêt de remarquer que les articles les plus critiques ont tous été publiés avant le milieu des années 90, c'est-à-dire avant le moment où les prix des logements vont commencer à augmenter très fortement. Vu d'aujourd'hui, et en prenant acte de l'évolution des prix sur ces dernières années en France, il semble particulièrement opportun de reprendre cette question. Plus largement, les questions relatives à la répartition et au transfert de richesse entre les générations présentent une actualité importante (D’ALBIS et MOOSA (2015)).

\footnotetext{
${ }^{2}$ En d'autres termes, le facteur g était omis.
} 


\section{Choix des variables démographiques pour le facteur d}

Pour la sphère démographique, les données mobilisées sont celles des recensements de la population réalisés par l'INSEE. Deux variables de niveau départemental et de fréquence annuelle sont utilisées : une variable absolue, la population totale notée TPOP, et une variable relative, le ratio de dépendance des personnes âgées, notée $\mathrm{OLDDEP}^{3}$. Cette grandeur mesure le degré de vieillissement de la population, en rapportant le nombre de personnes de plus de 60 ans à la population en âge de travailler (entre 20 à 59 ans). L'impact attendu sur le prix des logements est positif pour TPOP (plus la population augmente, plus la demande est forte, plus les prix augmentent), et négatif pour OLDDEP (comme cela a été discuté ci-dessus). Nous avons souhaité rajouter une seconde variable de structure, notée DIV $^{4}$ qui rend compte du taux de divorce. Cette variable est également de fréquence annuelle mais elle n'est disponible qu'à l'échelle nationale. L'impact attendu est positif: plus le taux de divorce augmente, plus la demande et le prix augmentent.

\section{d. Choix des variables économiques pour le facteur $\mathrm{g}$}

La variable venant immédiatement à l'esprit pour le facteur g est le PIB. Cependant, elle présente quelques défauts. Elle inclut en effet l'activité sectorielle de la production de logements et les activités de service associées, ce qui pourrait contribuer à accroître l'endogénéité de la variable à expliquer. D'autre part cet indicateur enregistre beaucoup d'informations relatives au contexte économique des entreprises. Si on est en droit de discuter du lien entre le prix des logements et la vie des entreprises, néanmoins ce lien n'est pas le plus direct et il nous éloigne un peu de la question considérée. L'information sur le PIB au niveau départemental corrigée de ces deux imperfections n'étant pas disponible, nous y substituons le revenu disponible des ménages, notée REV (source Oxford Economics), de niveau départemental et de fréquence annuelle. L'impact attendu est positif.

Nous utilisons également une variable relative aux conditions bancaires, le taux effectif global pour les prêts immobiliers à taux fixe, notée TEG, de niveau national et de fréquence annuelle. Le signe attendu pour cette variable est négatif : une baisse des taux d'intérêt diminue le coût du crédit pour les ménages, les solvabilise davantage, augmente la demande et donc les prix.

Des questions relatives à l'impact du système financier sur les marchés immobiliers (AALBERS, CHRISTOPHERS (2014)) pourraient aussi être considérées, comme cela a déjà été documenté pour les marchés de l'immobilier d'entreprise (HALBERT ET AL. (2014)). Toutefois, pour le cas qui nous occupe ici, à savoir l'immobilier résidentiel en France, la présence des investisseurs institutionnels est faible comme le souligne HALBERT (2013). Les ménages propriétaires, les particuliers bailleurs et les bailleurs sociaux constituent l'essentiel du marché. Cette situation est à relier au fait que le bien résidentiel est le moyen privilégié par les particuliers pour préparer leur retraite.

\footnotetext{
${ }^{3}$ Notation conforme à la littérature, OLDDEP signifiant 'Old dependency'.

${ }^{4}$ Caractéristiques de la nuptialité et des divorces depuis 1985, INED.
} 


\section{e. Spatialisation de l'étude}

En plus du facteur d et du facteur g, il est important d'avoir à l'esprit que le logement s'inscrit dans des territoires et que l'hétérogénéité spatiale doit être considérée.

L'emploi d'une technique de panel suppose que les observations sont repérées par deux dimensions, une dimension temporelle, et une dimension individuelle qui correspond ici aux départements. Les estimations de la relation économétrique seront réalisées globalement mais aussi en segmentant le panel afin d'apprécier la variabilité du mécanisme fondamental selon les différents espaces. Au niveau des variables explicatives, il est important de rappeler que les grandeurs démographiques (TPOP et OLDDEP) présentent elles aussi un fort caractère spatial. On parle ainsi parfois de la 'diagonale du vide' pour désigner une bande du territoire allant des Ardennes au Poitou qui, hormis pour quelques centres urbains, se caractérisent par une population faible, décroissante et vieillissante. La question des mobilités interdépartementales sera également prise en compte par un test d'autocorrélation spatiale. Le mouvement d'une partie des retraités vers les régions du Sud en est un exemple. Le test d'autocorrélation aura cependant pour objectif plus largement de contrôler des différents effets de dépendance entre les départements.

La question de l'intensité de la construction peut aussi se penser comme une variable spatiale ; Holland (1991) et Di PASQUALE et WHEATON (1994) ont pointé l'importance de cette variable. L'offre neuve de logements, notée OFF, de fréquence annuelle et de niveau départemental, est donc introduite - cette donnée est issue de Sit@del, base de données du ministère de l'Équipement, des Transports et du Logement. Le lien économétrique entre le marché du neuf et celui de l'ancien est en fait assez complexe, dans la mesure où une causalité bidirectionnelle peut exister. Apriori le coefficient attendu est négatif, c'est-à-dire que l'augmentation de l'activité de construction devrait conduire à une diminution des prix de l'immobilier ancien en raison de l'accroissement de l'offre. Mais il arrive assez souvent que la causalité soit inverse, car dans une logique de promoteurs la décision de construire est souvent une conséquence de la prospérité locale, et donc de l'augmentation du prix des logements anciens. La variable OFF est employée dans cette étude à deux titres: contrôler ces effets, mais également rendre compte des dynamiques locales des différents territoires.

\section{L'importance particulière du facteur d pour le prix des logements, premières intuitions}

a. Du baby-boom au papy-boom

Au cours des prochaines décennies, la population française va augmenter pour atteindre 70 millions d'habitants, la France sera alors le pays le plus peuplé d'Europe de l'Ouest. Le taux de fécondité étant modéré la population devrait cependant croitre à un rythme plus lent que celui observé pendant la $2^{\text {ème }}$ partie du $20^{\text {ème }}$ siècle. On peut se reporter à MAZUY ET AL. (2015) pour un bilan global de la situation démographique française. 
L'âge moyen va également augmenter. Avec $20 \%$ de sa population âgée de plus de 60 ans la France est aujourd'hui le $16^{\text {ème }}$ pays le plus vieux. Ce vieillissement de la population est la conséquence directe de la forte hausse de la natalité observée entre 1945 et 1970, appelée baby-boom. Le nombre d'enfants a avoisiné sur cette période les trois enfants, pour ensuite se stabiliser autour de deux (Figure 2). La taille d'une cohorte du baby-boom est donc en moyenne bien plus importante que pour une cohorte post baby-boom.

Figure 2 : Indicateur conjoncturel de fécondité

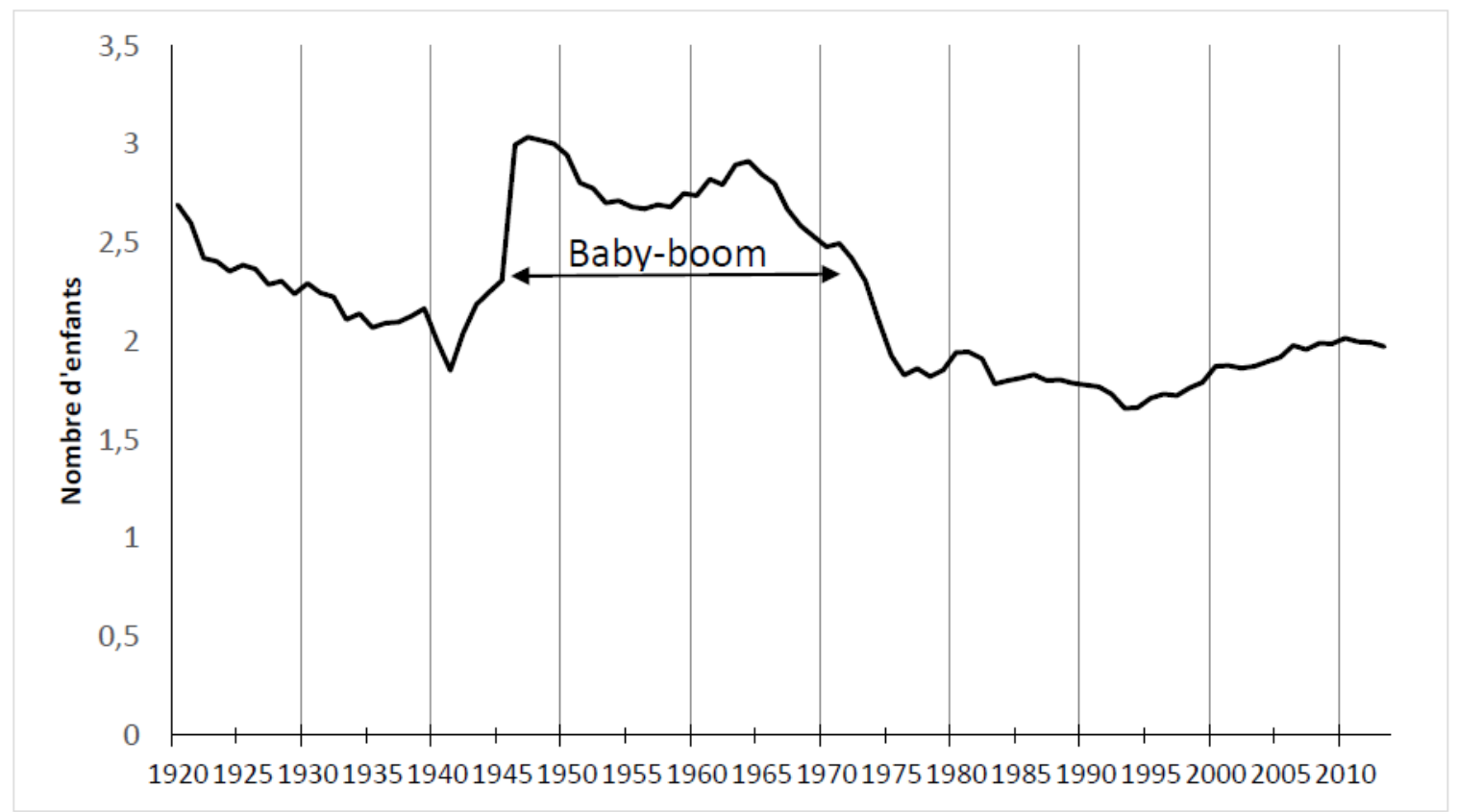

Indicateur conjoncturel de fécondité (INSEE), France métropolitaine, [1920 ; 2013]

Aujourd'hui ces classes d'âge arrivent à l'âge de la retraite. Il convient donc dorénavant de qualifier ce phénomène du terme de 'papy-boom'. On peut considérer qu'il débute en 2005-2006, moment où les individus nés en 1945 atteignent les 60 ans et commencent à partir en retraite. Cette tendance marquée va faire passer le nombre de seniors de 12,6 millions en 2005 à 22,3 millions en 2050 : une personne sur 3 ans aura plus de 60 ans, contre une sur 5 actuellement.

\section{b. Premières intuitions d'un lien entre le papy-boom et le prix des logements en France}

Le phénomène démographique majeur de la deuxième partie du $20^{\text {ème }}$ siècle consiste donc en cette bulle qui se translate au fur et à mesure des années. La question est alors de savoir quels sont les effets de cette distorsion générationnelle aux différents moments historiques. Par exemple, la fin des 30 glorieuses serait-elle à relier à l'arrivée sur le marché du travail des baby-boomers ? Et pour la question qui nous intéresse, peut-on trouver un lien entre la période du pré-papy-boom (19952005) et l'envolée des prix des logements, et un lien entre le papy-boom et la lente érosion des prix depuis 2006 ? 
Pour documenter cette question nous introduisons le ratio OLDDEP qui rapporte le nombre de personnes de plus de 60 ans, au nombre de personnes entre 20 et 60 ans. Cette mesure est utilisée dans beaucoup d'études empiriques et peut s'interpréter, à peu près, comme le nombre de retraités par actifs. Différentes recherches concluent que son impact est significatif et inverse sur les prix immobiliers. Qu'en est-il pour le cas de la France, temporellement et géographiquement?

La figure 3 met en rapport l'évolution du OLDDEP et les prix immobiliers (bruts, et nets de l'inflation), pour l'ensemble de la France, sur les 40 dernières années. Le lien semble effectivement exister. Ainsi, de 1975 à 1981 (période 1) le OLDDEP diminue alors que les prix du résidentiel augmente. Cette relation d'opposition se vérifie aussi de 1981 à 1986 (période 2). Par contre de 1986 à 1992 (période 3) il ne semble pas en aller ainsi, les prix immobiliers ont probablement alors été soumis à un autre facteur important. De 1992 à 1998 (période 4), le lien se vérifie à nouveau. Puis, de 1998 à 2006 (période 5 du pré papy-boom), on assiste à un ralentissement du OLDDEP alors que les prix augmentent fortement, laissant entrevoir que le lien est à considérer aussi en variations. Enfin, après 2006 (période 6, papy-boom), la relation d'opposition semble toujours bien présente : les prix ralentissent voire diminuent, alors que le ratio augmente très fortement. L'étude empirique de la section suivante portera sur ces deux dernières périodes.

Figure 3 : Ratio Old-dep et prix immobiliers

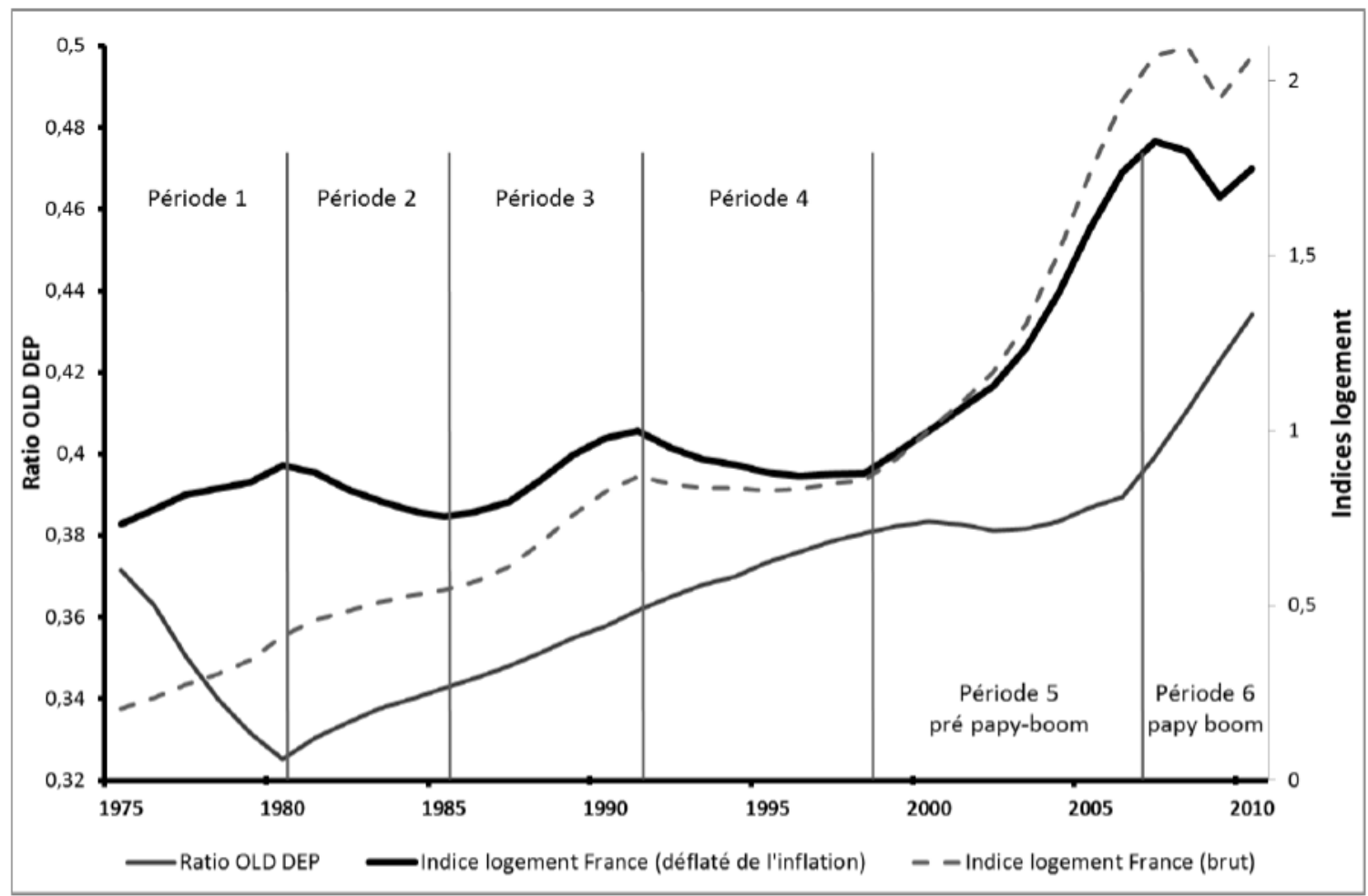

Ratio Old-Dep: nombre de personnes de plus de 60 ans / nombre de personnes entre 20 et 60 ans. Indices immobiliers brut et déflaté par I'IPC (indice des prix à la consommation). Période [1975; 2013]. France métropolitaine. Sources: CGEDD, INSEE. 
Il convient de relever le fait que cette relation est bien plus nette lorsque l'on considère les prix immobiliers déflatés de l'inflation, plutôt que les prix nominaux. Ainsi sur les périodes 3 et 4, la relation d'opposition entre le OLDDEP et les prix bruts n'apparait pas, alors qu'elle est très claire avec les prix nets ; invalidant au passage la réputation de protection contre l'inflation associée à l'immobilier.

Ce lien peut-il être observé à une échelle géographique plus fine et présente-t-il des spécificités ? La figure 4 fournit pour quatre départements les variations des prix des logements et celles du ratio OLDDEP, sur la période [2000; 2013]. Le lien inverse parait se manifester clairement, avec toutefois quelques nuances. Pour la Creuse, la relation d'opposition peut être observée d'une manière très pure, les deux courbes présentent une forte symétrie. Le lien est également net pour les Bouches-du-Rhône. Par contre pour les Alpes-Maritimes des facteurs complémentaires semblent agir, ainsi que pour Paris. Ces deux exceptions ont été retenus car elles sont affectées par des mouvements interdépartementaux de population: Paris attire des actifs en début ou milieu de carrière, tandis que la ville de Nice est attractive pour les retraités. Pour Paris, le facteur $g$ a probablement aussi un rôle renforcé. Enfin, il faut noter pour ces quatre graphiques (et pour tous les autres départements) le caractère de pivot des années 2005-2006 ; un croisement des courbes se réalise à cette date, on passe du pré papy-boom au papy-boom.

Figure 4 : Variations des prix immobiliers et du ratio Old-dep pour la Creuse, les AlpesMaritimes, les Bouches-du-Rhône et Paris
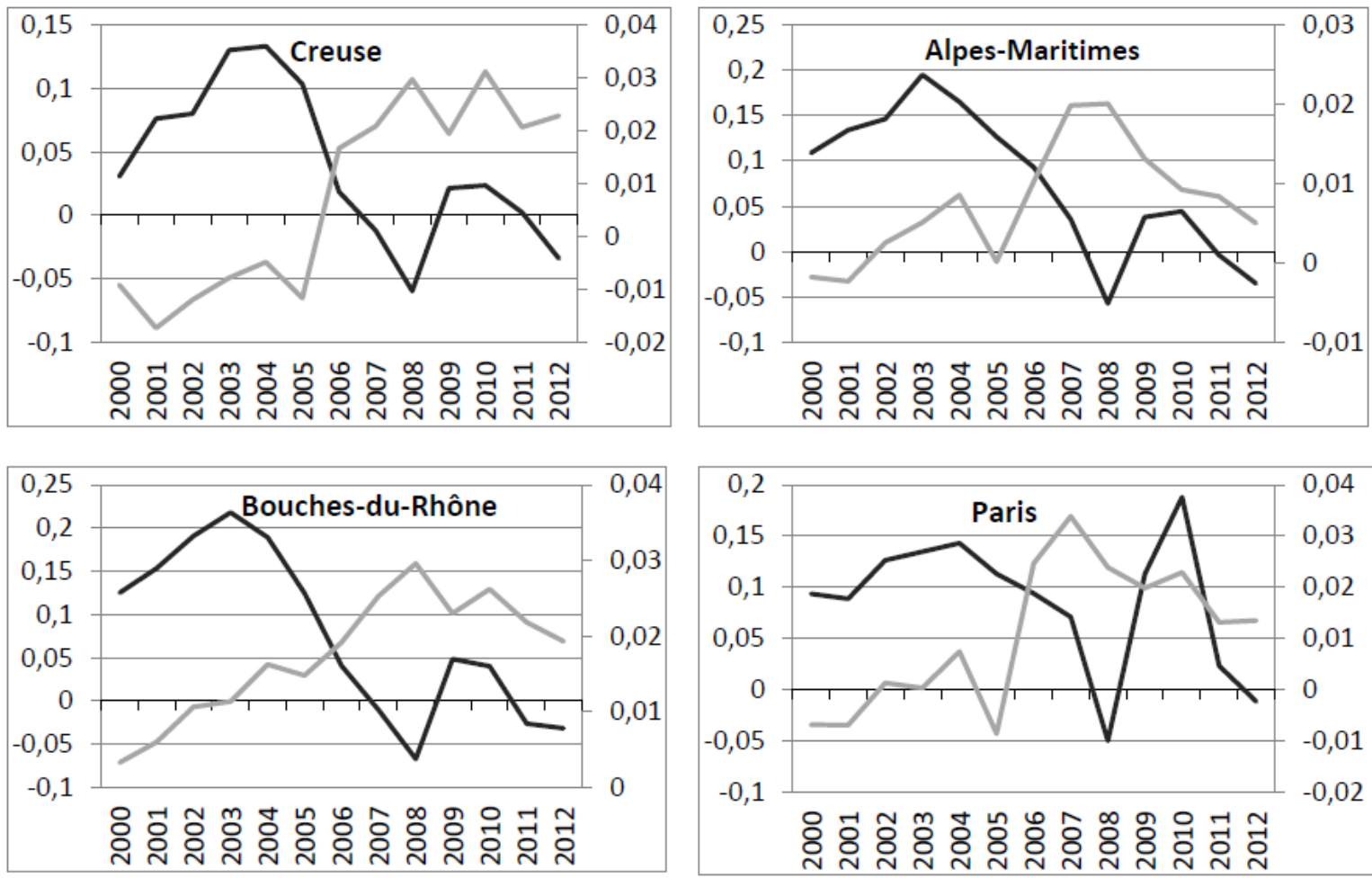

ـ : variation des prix immobiliers, déflatés de l'inflation par l'IPC (échelle de gauche)

— : variation du ratio OLD DEP (échelle de droite)

Période [2000 ; 2013]. Sources : INSEE, INSEE-Notaires, Immoprix. 


\section{Estimation d'un modèle de panel}

Les statistiques descriptives de la section précédente ont souligné l'importance du facteur démographique. La présente section vise à en quantifier les effets.

\section{a. Données de panel et variables}

Afin de répondre aux besoins de l'analyse et pour prendre en compte la double dimension, spatiale et temporelle du phénomène, nous avons recours à une approche en données de panel. Cette technique présente plusieurs avantages comparativement aux séries temporelles et aux coupes transversales. Les deux axes, individuel (ici géographique) et temporel, permettent d'accroître considérablement la taille de l'échantillon; ce qui a pour effet de réduire les problèmes de multicolinéarité et d'améliorer la précision des estimations. Cette approche permet aussi de contrôler la variabilité et l'hétérogénéité observée et inobservée des individus.

Notre panel est un panel court cylindré constitué de 94 départements de la France métropolitaine et de 14 observations annuelles, de 2000 à 2013, soit 1316 observations. Les indices hédoniques des logements anciens utilisés dans cette étude sont produits par l'INSEE et Immoprix, à partir des bases BIEN (Île-de-France) et Perval (Province). Une fois déflatés ${ }^{5}$, ils fournissent les variables à expliquer PAPP et PMAI, pour les prix des appartements et des maisons. Les variables explicatives ont été présentées ci-dessus. Pour rappel il s'agit, pour le facteur g des grandeurs REV ${ }^{6}$ et TEG, pour le facteur d des grandeurs TPOP, OLDEP et DIV, et pour le facteur spatial de la variable OFF. Les signes attendus sont indiqués dans la Figure 1.

\section{b. Tests préalables et choix du modèle}

\section{i. $\quad$ Causalité au sens de Granger, stationnarité et co-intégration}

La causalité au sens de Granger a d'abord été testée entre les prix immobiliers et la variable OLDDEP. Elle est significative et bidirectionnelle, OLDDEP cause les prix et inversement. Les tests de Harris, Tzvalis (1999) et d'Im, Pesaran, Shin ${ }^{7}$ (1997) permettent de retenir l'hypothèse que les séries présentent des racines unitaires en niveau mais qu'elles sont stationnaires en différence première, le modèle sera donc spécifié en variations. La co-intégration du modèle présenté cidessous a été testée avec les tests de Pedroni (2004) et n'a pas été validée. Le fait que le panel soit sur treize années avec une fréquence annuelle, que les séries soient peu volatiles, et que de plus elles manifestent des tendances assez persistantes nous confortent dans l'idée que la dissociation d'une tendance de long-terme et de dynamiques de court-terme n'est pas pertinente pour cette étude.

\footnotetext{
${ }^{5}$ Indices déflatés avec l'IPC (indice des prix à la consommation).

${ }^{6}$ Le revenu est exprimé en euros constants (source : Oxford Economics).

${ }^{7}$ Le choix des tests de racines unitaires HT et IPS s'est fait en référence à leur pertinence pour les panels courts présentant un grand nombre d'individus et une dimension temporelle courte $(\mathrm{N}>\mathrm{T})$.
} 


\section{ii. Méthode d'estimation et modèle retenu}

Lorsqu'on utilise les données de panel, il convient tout d'abord de tester la pertinence du recours à l'étude de plusieurs individus et donc de tester l'existence d'effets individuels. Ceci revient à considérer l'uniformité du modèle pour tous les départements (spécification homogène) versus l'existence de spécificités départementales (spécification hétérogène). Pour ce faire, l'équation est estimée en utilisant la régression à effets fixes qui fournit aussi le test d'existence des effets individuels. La p-value associée à la statistique de Fisher est inférieure à $1 \%$ pour les appartements et les maisons, validant la présence d'effets individuels. Il convient ensuite de les spécifier au regard de leur corrélation avec les autres variables ; s'il y a corrélation, alors l'estimation à effets fixes est plus pertinente que l'estimation à effets aléatoires, et inversement. La p-value associée à la statistique de Hausman étant inférieure à $1 \%$ pour les deux marchés, l'estimation à effets fixes est donc plus pertinente ${ }^{8}$. Nous retenons donc une spécification avec présence d'effets individuels avec une estimation à effets fixes.

Comme indiqué dans la revue de littérature le choix du modèle peut varier d'un pays à l'autre. Le modèle retenu pour le cas de la France est le suivant, inspiré du modèle de TAKATS (2012):

$$
\begin{aligned}
\Delta \ln \text { PAPP }_{\text {it }}(\text { ou } & \left.\Delta \ln \text { PMAI }_{i t}\right) \\
& =\alpha+\beta_{1} \Delta \ln \operatorname{REV}_{\text {it }}+\beta_{2} \Delta \ln \text { TOTPOP }_{\text {it }}+\beta_{3} \Delta \ln \text { OLDDEP }_{i t} \\
& +\beta_{4} \Delta \operatorname{lnOFF}(\text { AP ou MA })_{i t}+\beta_{5} \Delta \ln \text { TEG }_{t}+\beta_{6} \Delta \ln \operatorname{liV}_{\mathrm{t}}+v_{t}+\varepsilon_{\text {it }}
\end{aligned}
$$

La spécification est en différence de logarithmes afin d'obtenir des élasticités et de remédier à la non-stationnarité des variables en niveau. L'indice $i$ pointe les départements, l'indice $t$ pointe les années. Les résultats sont présentés dans le tableau 1. Les variables sont ajoutées une à une afin d'observer leur contribution au $\mathrm{R}^{2}$ ajusté. Le modèle complet est le M6. Pour le M7 les variables démographiques ont été retranchées.

\section{iii. Causalité inverse localement et autocorrélation spatiale}

La question d'un lien inverse entre le niveau des prix et le ratio OLDDEP peut aussi se poser. Un mouvement de prix à la hausse pourrait par exemple amener des mouvements migratoires de certaines classes d'âges, vers les départements limitrophes, et modifier localement les ratios de dépendance. L'influence de ces phénomènes sur les résultats a été testée en calculant un indice de Moran, qui rejette l'hypothèse d'une autocorrélation spatiale. La stabilité du coefficient OLDDEP obtenu avec les différentes segmentations (Tableau 2) est également un élément qui laisse penser que le lien prix-OLDDEP est estimé d'une manière robuste.

\footnotetext{
${ }^{8}$ Lorsque l'on dissocie l'effet temporel et l'effet individuel des variables, la plupart d'entre elles ont une variance 'within' plus importante qu'une variance 'between'. Une estimation à effets aléatoire conduirait à une importante perte d'information, confirmant la pertinence des effets fixes.
} 
Tableau 1 : Estimations des modèles appartements et maisons

\begin{tabular}{|c|c|c|c|c|c|c|c|c|}
\hline Appartements & & & & & & & & \\
\hline Fixed effect estimation & M1 & M2 & $\mathrm{M} 2^{\prime}$ & M3 & M4 & M5 & M6 & M7 \\
\hline$\Delta$ Ln OLDDEP & $-3,02^{* * *}$ & $-2,58^{* * *}$ & $-2,75^{* * *}$ & $-2,43^{* * *}$ & $-2,16^{* * *}$ & $-2,08^{* * *}$ & $-1,77^{* * *}$ & \\
\hline$\triangle \mathrm{Ln}$ TOTPOP & & $6,9^{* * *}$ & & $6,46^{* * *}$ & $7,05^{* * *}$ & $7,35^{* * *}$ & $5,11^{* * *}$ & \\
\hline$\Delta \mathrm{Ln}$ REV & & & $0,46^{* * *}$ & $0,30^{* * *}$ & $0,34^{* * *}$ & $0,36^{* * *}$ & $0,61^{* * *}$ & $1,58^{* * *}$ \\
\hline$\Delta \mathrm{Ln}$ OFF & & & & & $0,02^{* * *}$ & $0,02^{* * *}$ & $0,02^{* * *}$ & $0,03^{* * *}$ \\
\hline$\Delta \mathrm{Ln} T E G$ & & & & & & $-0,03^{* *}$ & 0,02 (n.s.) & $-0,01$ (n.s.) \\
\hline$\Delta$ Ln DIV & & & & & & & $0,29^{* * *}$ & $0,41^{* * *}$ \\
\hline c & $0,085^{* * *}$ & $0,041^{* * *}$ & $0,075^{* * *}$ & $0,037^{* * *}$ & $0,029^{* * *}$ & $0,024^{* * *}$ & $0,029^{* * *}$ & $0,016^{* * *}$ \\
\hline R2 ajusté & $37,99 \%$ & $41,91 \%$ & $39,00 \%$ & $42,29 \%$ & $46,13 \%$ & $46,33 \%$ & $49,78 \%$ & $34,81 \%$ \\
\hline Bilan Pedroni 1 & $3 / 11$ & $5 / 11$ & $8 / 11$ & $4 / 11$ & $5 / 11$ & $5 / 11$ & $5 / 11$ & $0 / 11$ \\
\hline Bilan Pedroni 2 & $5 / 11$ & $4 / 11$ & $6 / 11$ & $3 / 11$ & $5 / 11$ & $5 / 11$ & $5 / 11$ & $2 / 11$ \\
\hline Bilan Pedroni 3 & $3 / 11$ & $4 / 11$ & $3 / 11$ & $5 / 11$ & $6 / 11$ & $4 / 11$ & $3 / 11$ & $0 / 11$ \\
\hline
\end{tabular}

Estimations des modèles de panel, la variable à expliquer est la variation des prix des appartements déflatés par l'IPC. ${ }^{* * *},{ }^{* *}$ et ${ }^{*}$ désignent les significativités à $1 \%, 5 \%$ et $10 \%$. Pedroni 1,2 et 3 sont des tests de co-intégration respectivement : sans tendance déterministe, avec une tendance et un intercept déterministes, sans tendance ni intercept déterministes.

\begin{tabular}{|c|c|c|c|c|c|c|c|c|}
\hline Maisons & & & & & & & & \\
\hline Fixed effect estimation & M1 & $\mathrm{M} 2$ & M2' & M3 & M4 & M5 & M6 & M7 \\
\hline$\triangle$ Ln OLDDEP & $-3,26^{* * *}$ & $-2,85^{* * *}$ & $-2,87^{* * *}$ & $-2,58^{* * *}$ & $-2,07^{* * *}$ & $-2,11^{* * *}$ & $-1,93^{* * *}$ & \\
\hline$\triangle \mathrm{Ln}$ TOTPOP & & $6,33^{* * *}$ & & $5,55^{* * *}$ & $6,29^{* * *}$ & $6,12^{* * *}$ & $4,55^{* * *}$ & \\
\hline$\Delta \mathrm{Ln}$ REV & & & $0,66^{* * *}$ & $0,52^{* * *}$ & $0,51^{* * *}$ & $0,50^{* * *}$ & $0,73^{* * *}$ & $1,74^{* * *}$ \\
\hline$\Delta \mathrm{Ln}$ OFF & & & & & $0,12^{* * *}$ & $0,13^{* * *}$ & $0,13^{* * *}$ & $0,15^{* * *}$ \\
\hline$\triangle \mathrm{Ln} T E G$ & & & & & & 0,02 & $0,12^{* * *}$ & $0,09^{* * *}$ \\
\hline$\Delta$ Ln DIV & & & & & & & $0,27^{* * *}$ & $0,39^{* * *}$ \\
\hline $\mathrm{c}$ & $0,082^{* * *}$ & $0,042^{* * *}$ & $0,067^{* * *}$ & $0,034^{* * *}$ & $0,025^{* * *}$ & $0,024^{* * *}$ & $0,03^{* * *}$ & $0,011^{* * *}$ \\
\hline R2 ajusté & $46,04 \%$ & $49,46 \%$ & $48,25 \%$ & $50,78 \%$ & $60,47 \%$ & $60,52 \%$ & $62,78 \%$ & $45,76 \%$ \\
\hline Bilan Pedroni 1 & $5 / 11$ & $4 / 11$ & $8 / 11$ & $5 / 11$ & $5 / 11$ & $6 / 11$ & $6 / 11$ & $2 / 11$ \\
\hline Bilan Pedroni 2 & $5 / 11$ & $5 / 11$ & $6 / 11$ & $5 / 11$ & $5 / 11$ & $6 / 11$ & $6 / 11$ & $2 / 11$ \\
\hline Bilan Pedroni 3 & $0 / 11$ & $5 / 11$ & $3 / 11$ & $5 / 11$ & $4 / 11$ & $3 / 11$ & $3 / 11$ & $0 / 11$ \\
\hline
\end{tabular}

\section{c. Résultats et interprétations}

Les signes des coefficients correspondent globalement à ceux attendus. La taille de la population, le revenu et le taux de divorce impactent positivement les prix, le ratio OLDDEP négativement. L'effet du taux d'intérêt est instable et l'offre neuve est associée à un coefficient positif. Le $\mathrm{R}^{2}$ ajusté du modèle M6 est substantiel : 50\% pour les appartements et 63\% pour les maisons. Il est intéressant de constater que la variable OLDDEP contribue fortement au pouvoir explicatif de ce modèle ${ }^{9}$, et que l'inclusion progressive des autres variables n'améliore qu'assez faiblement le $\mathrm{R}^{2}$ ajusté.

\footnotetext{
${ }^{9}$ Les modèles M1, avec la seule variable OLDDEP, possèdent déjà des $\mathrm{R}^{2}$ ajustés de $38 \%$ (appartements) et $46 \%$
} (maisons). 
Intéressons-nous tout d'abord, avec le modèle M6, aux facteurs traditionnellement mis en avant. Une augmentation du revenu de $1 \%$ produit une hausse du prix des appartements de $0,61 \%$, et de 0,73\% pour les maisons. Cela est assez modéré et cohérent avec les résultats de TAKATS $(2012)^{10}$. Cette variable ne semble donc pas être en mesure de fournir une explication à la très forte hausse des prix observée entre 1998 et 2006. Deuxièmement, et contrairement à l'intuition, les taux semblent jouer un rôle tout à fait mineur, voire nul : les coefficients sont faibles, pas toujours significatifs et instables quant à leur signe. Troisièmement, la variable spatiale de l'offre neuve est positive et faible. Cela peut sembler contradictoire car on s'attendrait à ce que l'augmentation de l'offre produise une baisse des prix. Mais ce genre de résultat, apparemment paradoxal, est bien connu ; il est dû au fait que les promoteurs décident souvent de développer leurs projets dans des zones porteuses et haussières. L'effet direct de baisse des prix par augmentation de l'offre neuve est donc ici de deuxième ordre par rapport à l'effet inverse, causé par les stratégies de promotion. Cela signifie, en particulier, que les politiques de soutien à la construction neuve sont relativement sans effet sur les prix de l'ancien. Quatrièmement, le taux de divorce a un impact positif et faible. $\mathrm{Si}$ on prend le cas des appartements et que l'on considère que le taux de divorce augmente fortement en passant de $40 \%$ à $50 \%$, l'impact sur les prix ne serait alors que de $+6,68 \%$. Ainsi, contrairement à ce qui est souvent énoncé, le divorce impacte peu les prix. La séparation d'un ménage-propriétaire ne produit probablement pas deux propriétaires, mais un propriétaire et un locataire, voire même deux locataires étant donné l'augmentation des difficultés budgétaires de chacun.

Le modèle M7 ne prend pas en compte les variables démographiques. Son pouvoir explicatif est plus faible que pour le modèle M6, mais il reste substantiel. Il est toutefois remarquable de constater que l'inclusion de TOTPOP et de OLDDEP réduit fortement les coefficients des quatre autres variables. En d'autres termes, les facteurs classiquement mis en avant pour expliquer les dynamiques du prix des logements (revenu, taux d'intérêt, offre neuve, divorce) sont secondaires devant les déterminants démographiques. Cela a pour conséquence qu'une politique du logement visant à faire baisser les prix et/ou à solvabiliser la demande, et qui se baserait sur ces variables classiques serait vouée à l'échec.

L'impact principal sur les prix semble en fait être dû aux deux facteurs démographiques OLDDEP et TOTPOP. Une augmentation de $1 \%$ de la population totale fait augmenter les prix des appartements de $5,11 \%$, et de $4,55 \%$ pour les maisons. Quant au ratio OLDDEP, s'il passe de $40 \%$ à $41 \%$, les prix baissent de 4,28\% pour les appartements et de $4,65 \%$ pour les maisons. L'hypothèse selon laquelle l'évolution du nombre de retraités par actifs est un facteur de tout premier ordre pour expliquer le prix des logements trouve donc une validation par l'approche en données de panel, pour la France, sur la période [2000;2013]. Sur la base des prévisions démographiques, à l'horizon 2025 l'impact de la population totale sur les prix serait de $+23 \%$ pour les appartements et $+20 \%$ sur les maisons. L'impact du vieillissement serait de $-28 \%$ pour les appartements et $-31 \%$ sur les maisons ${ }^{12}$. Le marché devrait donc être baissier, légèrement pour les appartements $(-5 \%)$, et plus nettement pour les maisons $(-11 \%)$.

\footnotetext{
${ }^{10}$ Pour $1 \%$ d'augmentation du PIB, les prix immobiliers augmentent de $0,9 \%$ dans cet article.

${ }^{11} \operatorname{Exp}(0,29 * \operatorname{Ln}(50 / 40))-1$.

${ }^{12} \mathrm{TOTPOP}_{2015}=64,5 \mathrm{M}$ et OLDDEP $2015=0,48$. TOTPOP $_{2025}=67,2 \mathrm{M}$ et $\mathrm{OLDDEP}_{2025}=0,58$.
} 


\section{Analyse des spécificités territoriales}

Afin d'approfondir la connaissance de ce phénomène, nous re-estimons le modèle M6 suivant trois segmentations (Figure 5). La première distingue les départements jeunes, des départements âgés ${ }^{13}$ : Centre, Sud-Ouest, une partie du Sud-Est, quelques départements en Bretagne et en Normandie, ainsi que Paris. La seconde distingue les départements ruraux, des départements urbains ${ }^{14}$ : Ile-deFrance élargie, départements des métropoles régionales, à l'exception notable de la Gironde et de Bordeaux. La troisième segmentation est réalisée sur la base des quartiles de prix appartements et maisons. On y distingue clairement l'influence de Paris, la diagonale du vide ainsi que l'effet littoral. Celui-ci est très net pour les appartements ; pour les maisons il conviendrait plutôt de parler d'un effet Sud-Est. Ces segmentations présentent des structures spatiales hétérogènes qui ne sont pas équivalentes entre elles.

Tableau 2 : Estimations du modèle M6 selon les segmentations départementales jeune/âgés, urbain/rural et par quartiles de prix

\begin{tabular}{|c|c|c|c|c|c|c|c|c|}
\hline Variables & $\begin{array}{c}\text { AP } \\
\text { DEP } \\
\text { jeunes }\end{array}$ & $\begin{array}{c}\text { AP } \\
\text { DEP } \\
\text { vieux }\end{array}$ & $\begin{array}{c}\text { MA } \\
\text { DEP } \\
\text { jeunes }\end{array}$ & $\begin{array}{c}\text { MA } \\
\text { DEP } \\
\text { vieux }\end{array}$ & $\begin{array}{c}\text { AP } \\
\text { DEP } \\
\text { urbains }\end{array}$ & $\begin{array}{c}\text { AP } \\
\text { DEP } \\
\text { ruraux }\end{array}$ & $\begin{array}{c}\text { MA } \\
\text { DEP } \\
\text { urbains }\end{array}$ & $\begin{array}{c}\text { MA } \\
\text { DEP } \\
\text { ruraux }\end{array}$ \\
\hline REV & 0.42 & $0.85^{* * *}$ & $0.51^{\cdots *}$ & $0.99^{* * *}$ & 0.19 & $0.78^{* * *}$ & $0.32^{\circ}$ & $0.87^{* * *}$ \\
\hline TOTPOP & $7.12^{\cdots *}$ & $3.18^{* * *}$ & $5.69^{\cdots}$ & $3.52^{* *}$ & $7.41^{\cdots * *}$ & $3.90^{* * *}$ & $6.10^{\cdots *}$ & $3.91^{* * *}$ \\
\hline OLDDEP & $-1.67^{* * *}$ & $-1.86^{* *}$ & $-2.04^{* *}$ & $-1.78^{* *}$ & $-1.84^{* *}$ & $-1.74^{\cdots *}$ & $-2.27^{* *}$ & $-1.81^{\cdots}$ \\
\hline TEG & -0.04 & -0.02 & $0.14^{* *}$ & 0.10 & $0.04^{* *}$ & -0.02 & $0.17^{\cdots *}$ & $0.11^{* * *}$ \\
\hline OFF_AP ou MA & $0.04^{* *}$ & $0.01^{* * *}$ & $0.12^{\cdots *}$ & $0.13^{* *}$ & $0.05^{* * *}$ & $0.01^{* * *}$ & $0.12^{\cdots *}$ & $0.13^{* *}$ \\
\hline Tx Div & $0.26^{* *}$ & $0,34^{\cdots * *}$ & $0.25^{\cdots *}$ & $0.30^{* *}$ & $0.29^{* *}$ & $0.30^{* * *}$ & $0.27^{* *}$ & $0.28^{* * *}$ \\
\hline$\alpha$ & $0.02^{* *}$ & $0.03^{* * *}$ & $0.03^{\cdots}$ & $0.02^{* *}$ & 0.02 & $0.03^{* *}$ & 0.03 & $0.03^{* * *}$ \\
\hline \multirow[t]{2}{*}{$\mathrm{R}^{2}$ ajusté } & 0.45 & 0.55 & 0.54 & 0.71 & 0.48 & 0.51 & 0.50 & 0.66 \\
\hline & AP Q1 & AP Q2 & AP Q3 & AP Q4 & MA Q1 & MA Q2 & MA Q3 & MA Q4 \\
\hline REV & $0.89^{* * *}$ & $0.78^{* * *}$ & $0.67^{\circ \cdot}$ & $0.35^{*}$ & $0.78^{* * *}$ & $1.12^{* * *}$ & $0.50^{\cdots *}$ & $0.58^{* * *}$ \\
\hline TOTPOP & 0.90 & 0.89 & $5.84^{\cdots}$ & $9.23^{* *}$ & 2.20 & 0.37 & $6.11^{\cdots}$ & $6.18^{* * *}$ \\
\hline OLDDEP & $-1.59^{* *}$ & $-2.07^{* *}$ & $-1.79^{* *}$ & $-1.69^{* * *}$ & $-1.74^{\cdots}$ & $-1.70^{\cdots *}$ & $-2.18^{* *}$ & $-2.23^{\cdots}$ \\
\hline TEG & 0.06 & 0.01 & 0.03 & $0.10^{* *}$ & $-0.09^{*}$ & $0.18^{* * *}$ & $0.16^{* *}$ & $0.11^{* *}$ \\
\hline OFF_AP ou MA & $0.01^{* *}$ & $0.01^{*}$ & $0.03^{\cdots}$ & $0.07^{* * *}$ & $0.12^{\cdots *}$ & $0.15^{* * *}$ & $0.18^{\cdots *}$ & $0.09^{* * *}$ \\
\hline Tx Div & $0.31^{\cdots}$ & $0.30^{* * *}$ & $0.25^{\cdots}$ & $0.37^{* *}$ & $0.25^{\cdots *}$ & $0.35^{* *}$ & $0.23^{\cdots}$ & $0.30^{* * *}$ \\
\hline$\alpha$ & $0.04^{* *}$ & $0.04^{* * *}$ & 0.02 & -0.01 & $0.04^{\cdots *}$ & $0.04^{* *}$ & $0.02^{*}$ & 0.00 \\
\hline$R^{2}$ ajusté & 0.49 & 0.49 & 0.50 & 0.57 & 0.62 & 0.69 & 0.66 & 0.57 \\
\hline
\end{tabular}

Estimations du modèle de panel M6 sur les différents segments. Les variables à expliquer sont les variations des prix des maisons et des appartements, déflatés par I'IPC. ***, ** et * désignent les significativités à 1\%, $5 \%$ et $10 \%$. Segmentation 1 : départements jeunes (48) / départements âgés (46). Segmentation 2 : départements urbains (23) / départements ruraux (69). Segmentation 3 : par quartiles de prix, du moins cher Q1 au plus cher Q4.

\footnotetext{
${ }^{13}$ Cette segmentation est fonction de l'âge médian du département par rapport à l'âge médian de la population globale : 48 départements jeunes, 46 départements vieux.

${ }^{14}$ Segmentation définie par l'INSEE : 23 départements urbains, 69 départements ruraux.
} 
Les résultats sont présentés dans le tableau 2. Le pouvoir explicatif du modèle reste substantiel pour tous les segments. Les coefficients associés aux différentes variables et leur degré de significativité sont assez proches du modèle global. La manière dont la variable OLDDEP influence les prix est une chose très stable; si la distribution spatiale de cette donnée est hétérogène, pour autant son principe causal est homogène. Pour les autres variables, les causalités varient davantage.

Ainsi, pour le revenu (REV) et la population totale (TOTPOP), les effets différent entre les zones jeunes, urbaines ou chères, et les zones âgées, rurales ou peu chères. Pour les espaces du premier type la population totale a un rôle accru, le revenu impactant peu, voire pas, le prix des logements. A l'inverse, pour les zones du second type, la causalité du revenu est renforcée tandis que la population totale joue un rôle mineur, voire nul. Dans les zones jeunes-urbaines-chères le déterminant démographique prime sur le déterminant économique ; alors que pour les zones âgéesrurales-abordables c'est le déterminant économique qui prime.

Pour les taux (TEG) et l'offre neuve (OFF), la segmentation qui s'impose n'est pas territoriale mais basée sur le type de bien. Pour les appartements, les effets des taux et de la construction sont souvent non significatifs ou très faibles. Pour les maisons les effets sont positifs, tout en restant modestes; ils sont de plus contre-intuitifs. Pour le TEG, cela pourrait s'expliquer par un effet temporaire d'incitation : les taux étant en train de remonter, l'achat d'une maison étant de plus très engageant financièrement, il faudrait se hâter d'acheter, poussant ainsi les prix à la hausse. Pour la variable $\mathrm{OFF}$, les résultats semblent indiquer que la stratégie de promotion visant à construire dans les marchés haussiers vaut surtout pour le marché des maisons. Ces deux variables ont cependant des effets minimes sur les prix. Enfin, pour la variable divorce (DIV), on ne note pas de variabilité géographique de la causalité. 
Figure 5 : Segmentations spatiales

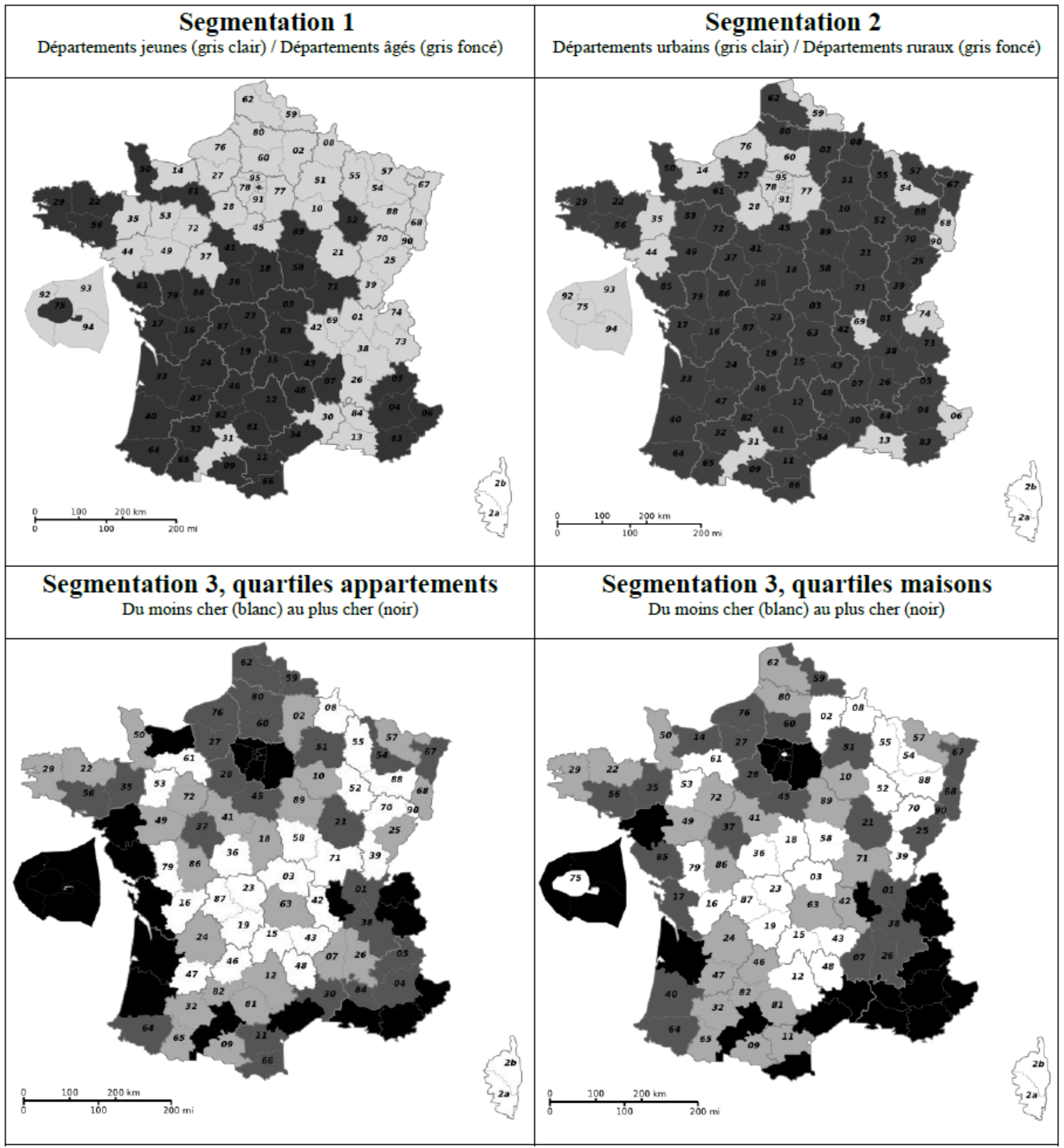

Segmentation 1: départements jeunes / départements âgés. Cette segmentation est fonction de l'âge médian du département par rapport à l'âge médian de la population globale. 48 départements jeunes, 46 départements âgés. Source INSEE, 2013.

Segmentation 2: départements urbains / départements ruraux. Segmentation définie par 1'INSEE. 23 départements urbains, 69 départements ruraux.

Segmentation 3 : par quartiles de prix, appartements et maisons. Sources : Notaires-INSEE, Immoprix, 2013. La Corse et Paris pour les prix maisons ne sont pas représentés en raison de données insuffisantes. 


\section{Le logement et l'accroissement de l'inégalité intergénérationnelle}

\section{a. Les trois temps de l'impact des baby-boomers sur les prix immobiliers en France}

L'identification de la structure démographique comme facteur de premier plan permet de mieux comprendre l'évolution récente et à venir des prix du logement. LE BAYON ET AL.(2013) indiquent que les principaux acheteurs de résidence principale en France seraient les 30-50 ans ${ }^{15}$. Sur cette base, on peut distinguer trois phases qui correspondent au déplacement et au vieillissement des cohortes du baby-boom.

La première phase est celle du pré papy-boom, elle se caractérise par la très forte hausse des prix entre 1996 et $2006^{16}$. C'est le moment où l'on trouve le plus grand nombre de baby-boomers entre 30 et 50 ans. Ces classes d'âges sont nombreuses et massivement acheteuses, cette envolée des prix correspond à un phénomène de demande. La seconde phase débute en 2006, année où les personnes nées dans l'immédiat après-guerre commencent à arriver à l'âge de la retraite. Il est notable que l'inflexion des prix coïncide précisément avec le début du papy-boom - il s'agit ici aussi d'un élément de confirmation du lien causal. Les 30-50 ans étant moins nombreux dans la population, l'intensité des achats diminue, les prix des logements baissent peu à peu. Les volumes confirment ce fléchissement de la demande : le nombre de transactions a diminué de $10 \%$ par rapport à la première période (CGEDD d'après DGFiP (MEDOC) et bases notariales). Pour la troisième phase, qui débute actuellement, un autre phénomène vient s'y adjoindre : une augmentation régulière du nombre des décès est en effet à l'œuvre. De 530000 décès/an en moyenne sur les 10 dernières années, ce nombre devrait passer progressivement à 600000 en 2020, puis continuer à augmenter (INSEE). Les biens hérités sont pour une part conservés par les descendants, mais une fraction significative est aussi remise sur le marché, accroissant l'offre. Erosion de la demande et augmentation de l'offre devraient alors conduire à une poursuite de la baisse des prix.

Il semble en fait exister différents types d'ajustements immobiliers. Pour le Japon et l'Allemagne, pays les plus avancés dans le vieillissement, la baisse a été lente et régulière, quelques pourcents chaque année. Si l'on raisonne en prix immobiliers nets, ils ont baissé de $30 \%$ en Allemagne sur les vingt dernières années et de près de $50 \%$ pour le Japon en vingt-cinq ans (TAKATS (2012)). Pour les Etats-Unis la situation est différente. Suite aux problèmes sur les prêts subprimes le marché résidentiel a vu ses prix baisser rapidement, - 23\% en quatre ans, de 2007 à 2011 (indice CaseShiller national). Cette correction a été très brutale, mais on peut aujourd'hui considérer que la forte valorisation des propriétés, du point de vue des jeunes générations, relève du passé ${ }^{17}$. Pour le cas de la France, la question se pose donc de savoir quel sera le chemin suivi : une lente érosion,

\footnotetext{
15 Comme l'indique la Figure 6, on semble cependant assister depuis une dizaine d'années à un glissement de la propriété vers les cohortes les plus âgées, et des achats plus tardifs. Ainsi, en 2015, le taux de propriétaire chez les 5059 ans était tout juste au niveau de la moyenne nationale.

${ }^{16}$ Suivant les secteurs et les segments du marché, la hausse peut se poursuivre jusqu'en 2010. Nous retenons toutefois 2006 comme une date symbolique marquant le début du papy-boom.

${ }^{17}$ La remontée significative des indices immobiliers américains sur la période 2011-2015 se fait dans un contexte différent, elle s'accompagne en effet d'une évolution positive des revenus. Lorsque l'on rapporte les prix immobiliers au revenu disponible, la situation actuelle ne présente plus le même décrochage que celui qui prévalait sur la période 2000-2007 (Source CGEDD, Comparaison internationale, indice du prix des logements rapporté au revenu disponible par ménage).
} 
que l'on pourra qualifier du mot de 'déflation', mais qui ne sera en fait qu'un synonyme pour le mot 'papy-boom', ou bien un événement de type 'crise'.

\section{b. Concurrence générationnelle pour la propriété du logement}

Les générations ne sont pas en séquence, elles sont imbriquées les unes dans les autres. Il y a donc, de fait, une concurrence intergénérationnelle sur certains biens communs et en particulier pour le logement. Lorsque des phénomènes viennent affecter les effectifs des classes d'âge on peut donc s'attendre à ce qu'ils affectent aussi le rapport de force entre ces classes. L'idée d'une 'génération sacrifiée' est ainsi parfois mentionné lorsque l'on évoque la situation japonaise de ces vingt dernières années. Avec ses taux bas et son risque déflationniste, ce pays représente un cas d'étude particulièrement intéressant ${ }^{18}$ pour l'Europe vieillissante. L'adjectif 'sacrifiée', est très fort. Peutêtre suffirait-il de parler d'inégalité intergénérationnelle pour le cas de la France ? YATES (2011) a proposé d'introduire le concept de 'soutenabilité immobilière' dans ces réflexions en le définissant de la manière suivante :

"La soutenabilité immobilière signifie que les besoins d'une génération peuvent être satisfaits sans compromettre la capacité des générations futures à satisfaire les leurs. La soutenabilité s'oppose au stress immobilier, défini comme une situation où les ménages consacrent une part excessive de leurs revenus au logement. »

Même pour un pays comme les Etats-Unis, qui n'est pourtant pas le plus affecté par le vieillissement, ce débat semble pertinent. Lorsque l'on étudie l'évolution des classes d'âge, à l'intérieur de la population des propriétaires américains (US Census Bureau), on peut observer que depuis le milieu des années 90 la part des propriétaires de moins de 45 ans a fortement baissé, alors que celle des plus de 55 ans augmentait nettement. Le développement du marché des subprimes entre 2003 et 2007 mériterait d'être interrogé dans cette optique. Ces produits pourraient-ils être symptomatiques d'une concurrence générationnelle problématique et d'une éviction des jeunes générations de l'accès à la propriété ?

Pour le cas de la France, si l'on utilise le ratio revenu-disponible/prix-immobiliers (CGEDD) pour estimer le stress immobilier, il semble clair que les classes d'âge post baby-boom subissent, aujourd'hui, un bien plus grand stress que leurs ainés hier. La Figure 6 permet de préciser cela en considérant l'évolution de la structure de répartition de la propriété immobilière en fonction de l'âge. Les taux de propriétaires des différentes classes d'âge sont présentés pour les années 1998, 2004, 2010 et 2015 (Enquêtes Patrimoine, INSEE). Ces taux ont été normalisés avec le taux global de propriétaires l'année considérée. Un ratio supérieur (inférieur) à 1 indique que la classe d'âge est plus (moins) propriétaire que l'ensemble de la population. Pour les résidences principales la forme patrimoniale classique en crochet, croissance assez régulière puis diminution sensible après 70 ans $^{19}$, s'observe en 1998 et 2004. Mais avec le début du papy-boom en 2006 cette structure se transforme en une courbe strictement croissante. Dans le même temps la classe d'âge des 50-59 ans, qui était la plus propriétaire, voit son taux diminuer fortement, à peu près au niveau de la moyenne nationale; ce phénomène est aussi observable pour les 40-49 ans. Pour les autres

\footnotetext{
18 cf. LANGUILLON-AUSSEL (2015) pour le marché des bureaux par exemple.

${ }^{19}$ Cette baisse est à relier à différents phénomènes : maisons de retraite, résidences-services pour personnes âgées, prise en charge par les enfants...
} 
logements (résidences secondaires, investissements locatifs...), un changement structurel est aussi à l'œuvre ; la classe d'âge la plus propriétaire est aujourd'hui celle des 60-69 ans et plus celle des 50-59 ans. Il convient de rappeler deux éléments supplémentaires : le taux de propriétaires chez les retraités est estimé à $75 \%$ (INSEE), et à Paris $80 \%$ des appartements seraient détenus par des personnes de plus de 60 ans (EL KAROUI (2013)). Cette déformation de la structure de propriété s'est réalisée en un temps trop court pour être due à l'allongement de l'espérance de vie, elle est en fait la signature du papy-boom. Comme pour les Etats-Unis, la France vit donc un changement majeur dans la répartition de la propriété immobilière en faveur des plus de $60 \mathrm{ans}^{\mathbf{2 0}}$. Et il ne serait sans doute pas impossible de défendre l'idée selon laquelle cette concurrence générationnelle pour le logement serait en fait bien plus forte en France qu'outre-Atlantique, aujourd'hui.

Figure 6 : Structure par classes d’âge du taux de propriétaires normalisés en France, de 1998 à 2015

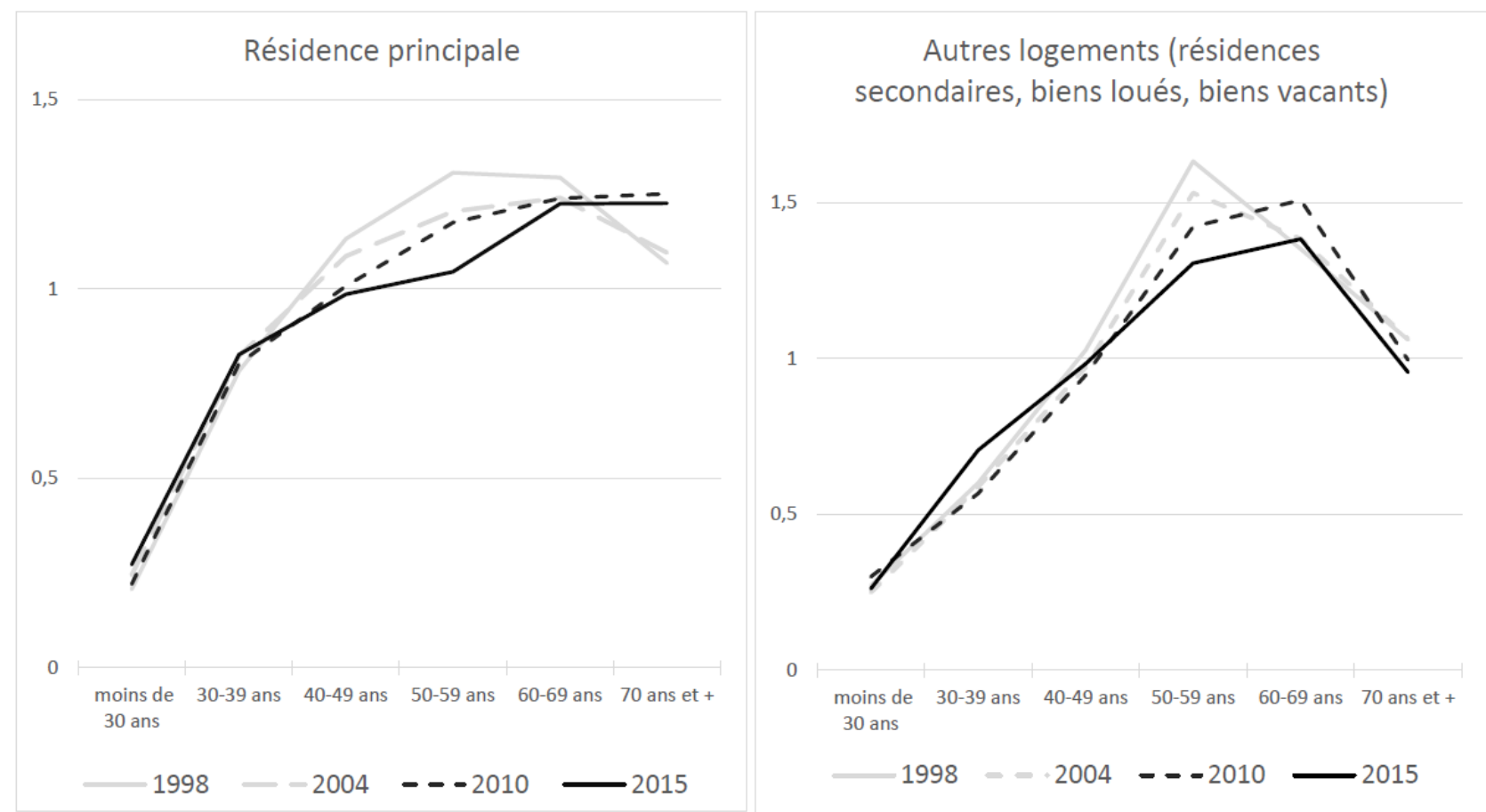

Pour chaque année, le taux de propriétaires de la classe d'âge est divisé par le taux de propriétaires global. Années : 1998, 2004, 2010, 2015. France métropolitaine. Sources : Enquêtes patrimoine, INSEE.

\footnotetext{
${ }^{20}$ La publication de l'ANIL, Habitat actualités, de juillet 2012 va aussi dans ce sens : «L'augmentation de la part des ménages âgés explique en grande partie la progression du taux de propriétaires occupants », ceci sur la base des Enquêtes Logement de 1984, 1988, 1992, 1996, 2001 et 2006.
} 


\section{c. Une politique du logement générationnellement orientée}

Il n'est donc pas impossible de trouver des explications à la très forte hausse des prix immobiliers et nous pensons que la donnée démographique est le facteur principal. Il n'est cependant pas exclu que ses effets puissent se trouver renforcés par des phénomènes de liquidité très (trop?) abondante, et par des facilités de crédit sur certaines périodes.

Lorsque les prix des logements sont multipliés par 2,5 en 10 ans, la question ne manque pas de venir dans le champ du débat public. Cette section discute les «solutions » habituellement mises en avant. Le soutien à la construction, avec des objectifs annuels énoncés, est l'une d'elles. Mais il convient d'indiquer que si l'on espère de ce soutien une baisse des prix, on se trompe dans les ordres de grandeur d'un facteur 10. Les distorsions entre les classes d'âges sont en effet de l'ordre du million, alors que les efforts de construction supplémentaires ne peuvent être que de quelques dizaines de milliers d'unités. La question de faire passer le nombre de constructions annuelles de 300000 à 400 000, ou 500 000, est d'importance secondaire. Cette inexactitude pourrait être mineure si la focalisation du débat sur la construction ne servait pas de paravent, empêchant de voir l'ampleur de la question démographique contemporaine.

Parmi ces «solutions » il faut également considérer le PTZ (prêt à taux-zéro), les aides à l'investissement locatif et les APL (aides personnalisées au logement). Elles sont aujourd'hui particulièrement discutables. La littérature économique suspecte que ces dispositifs produisent des effets inflationnistes indésirables, c'est-à-dire qu'ils contribuent à la hausse des prix (BONO et TRANNOY (2012), BEAUBRUN-Diant, MAURY (2015)), voire à la hausse des loyers pour le cas des APL. Ces avantages, qu'ils soient financiers ou fiscaux, seraient de plus partiellement captés par des acteurs à qui ils ne sont pas destinés.

C'est probablement le soutien à l'investissement locatif qui est l'exemple le plus net permettant de suspecter une politique générationnelle orientée pro-papy-boomers. Ces derniers sont en effet souvent propriétaires de leur résidence principale et n'ont plus de crédit à rembourser ${ }^{21}$. Leur capacité d'emprunt peut alors être employée pour l'investissement locatif car, en plus de la subvention fiscale des différents dispositifs, ils sont souvent de bons risques d'un point de vue bancaire. La question se pose en effet aussi en termes d'orientation des crédits bancaires. Aujourd'hui, les encours destinés à l'investissement locatif, soit $20 \%$ du total des encours immobiliers, représentent autant que les encours destinés à la primo-accession (données Banque de France). L'enquête Bailleurs-2015 du site PAP.fr indiquait également que 60\% des bailleurs ont plus de 50 ans.

Si l'on étudie la question du locataire et du loyer, d'un point de vue financier, la situation est en fait très favorable aux propriétaires. Les jeunes générations, c'est-à-dire les personnes qui auraient pu acheter dans une situation standard, fournissent un vivier important de locataires solvables. A titre d'exemple, seul $18 \%$ des ménages d'Île-de-France entre 25 et 45 ans pouvaient acheter un bien immobilier correspondant à leurs besoins en 2013 (Baromètre CAPACIM). L'effet inflationniste des APL sur les loyers assure aux propriétaires une rentabilité locative très confortable : 5,91\% pour la Seine Saint-Denis en $2016^{22}$ (MeilleursAgents), et même davantage

\footnotetext{
${ }^{21}$ Ce statut dit de 'propriétaire non accédant' est d'ailleurs celui de $70 \%$ des propriétaires, souvent âgés (INSEE, Compte du logement 2011).

${ }^{22}$ Chiffre obtenu en rapportant le loyer moyen annuel au $\mathrm{m}^{2}$ (184€), au prix moyen au $\mathrm{m}^{2}$ (3114€), en janvier 2016.
} 
pour les résidences étudiantes. On peut même aller jusqu'à s'interroger sur le fait de savoir s'il ne s'agirait pas là, purement et simplement, d'un transfert de richesse de l'Etat aux bailleurs du parc locatif privé ?

L'alternative pour les jeunes générations consiste souvent entre la location dans les centres urbains et l'achat dans le péri-urbain. Mais même l'achat peut aujourd'hui être interrogé. Car si ce mouvement de baisse des prix se poursuit sur les quinze prochaines années du papy-boom, quelle sera la création de valeur patrimoniale pour ces personnes ? Ils auront acheté très cher un bien, en le finançant par de la dette, alors même que ce bien risque de perdre une partie de sa valeur. En ce qui concerne les papy-boomers, l'argument de la transmission du patrimoine à leurs enfants ne saurait suffire. Car étant donnée l'espérance de vie lorsque cette transmission se produit la génération qui en hérite approche déjà de sa retraite : l'âge moyen des héritiers en France est de 52 ans (source Notaires de France). Ces différentes réflexions mettent l'accent plus globalement sur la question de l'accroissement des inégalités entre les générations (EL KAROUI (2013)).

\section{Conclusion}

Pour étudier l'effet du facteur démographique sur le prix des logements et leur si curieuse dynamique depuis quinze ans, nous nous sommes inscrits dans un modèle à trois dimensions. Il apparait à l'issue de cette étude que la sphère économique, approximée par les variables du revenu et des taux d'intérêt, est tout à fait secondaire devant la sphère démographique, approximée par la population totale et par le ratio entre le nombre de retraités et la population active. Il est de plus notable que le divorce a un rôle mineur, et que les variations de la construction neuve n'impactent pas les prix de l'ancien. La déclinaison spatiale du phénomène nous a appris que la causalité entre prix et vieillissement est spatialement assez homogène. De plus, pour les zones urbaines-jeuneschères, le facteur démographique de la population totale prime sur le revenu, alors que pour les départements ruraux-âgés-abordables la situation est inverse.

La question du lien entre structure démographique de la population et prix immobiliers, que la littérature suggère, trouve donc dans le cas de la France une réponse positive. En nous basant sur ces résultats nous avons poursuivi la réflexion autour du thème de la concurrence générationnelle, concurrence forcément insoutenable par les jeunes générations, en identifiant des éléments d'une captation accrue de la propriété par les retraités. Une lecture de la politique du logement comme étant pro-papy-boomers en a découlé.

On peut estimer la valeur du parc immobilier français aujourd'hui à 6363 milliards d'euros ${ }^{23}$. En ramenant cette valeur en 1998, sur la base de l'indice Notaires INSEE France ${ }^{24}$, ce même parc ne valait alors que 2657 milliards d'euros. La plus-value captée par le parc résidentiel est donc de 3706 milliards d'euros ! On ne saurait considérer que ce très important enrichissement ne compte

\footnotetext{
${ }^{23}$ Les comptes du logement 2011 indiquent que le nombre d'unités d'habitation est de 34 millions. 56\% sont des maisons avec une surface moyenne de $102 \mathrm{~m}^{2}, 44 \%$ des appartements avec une surface moyenne de $62 \mathrm{~m}^{2}$. En janvier 2016, MeilleursAgents indique le prix moyen au $\mathrm{m}^{2}$ en France est de 3077 euros pour les appartements, 1807 euros pour les maisons. La valeur du parc est donc : $34000000 *(0,56 * 102 * 1807+0,44 * 62 * 3077)=6363$ milliards d'euros. ${ }^{24} \mathrm{~L}$ 'indice vaut au T1-1998T1 : 42,8 et au T3-2015 : 103. En appliquant proportionnellement cette variation au parc on obtient $6363 *(43 / 103)=2657$ milliards d'euros. Le parc étant un peu plus petit en 1998 qu'en 2015, il s'agit d'une estimation haute de la valeur du parc en 1998.
} 
pas, qu'il serait une sorte de hors-bilan ; particulièrement quand ce hors-bilan est réparti d'une manière très inégalitaire suivant l'âge, et que cette inégalité s'est significativement renforcée depuis 15 ans. Cela, d'autant plus, qu'il existe des moyens efficaces permettant la mobilisation de ces richesses captives, immobilisées dans l'immobilier. Ces moyens peuvent être traditionnels comme le viager, ou plus financiers comme le viager hypothécaire (SHAN (2012)).

Cette situation interroge aussi le système des retraites sur le plan de l'équité générationnelle. Estil juste de verser des retraites à taux plein à des retraités propriétaires de leur résidence principale, sans prêt associé, parfois propriétaires d'une résidence secondaire et d'investissements locatifs, fiscalement aidés, alors même qu'ils pourraient extraire beaucoup de richesse de leur patrimoine immobilier ? La politique fiscale doit-elle se faire en direction du PTZ et de l'investissement locatif, ou prendre pour objectif d'accompagner et de favoriser la mobilisation de la valeur immobilisée dans l'actif immobilier afin de diminuer la charge importante qui pèse sur les jeunes générations ? L'inévitable augmentation du nombre de successions dans les années à venir ${ }^{25}$ pose aussi des questions sur l'imposition de ces héritages. Rappelons que la dette de l'Etat français est aujourd'hui de 2000 milliards d'euros, soit juste la moitié de la plus-value enregistrée par le parc immobilier en quinze ans. Les ordres de grandeur sont comparables. La légitimité de cette plusvalue, non-productive et rentière, doit être questionnée et en particulier lors des transmissions. Il y a là de puissants moyens pour réduire la dette de l'Etat...

Le logement est emblématique de l'accroissement des inégalités intergénérationnelles. La question de la distribution des richesses ne peut plus aujourd'hui être posée uniquement en des termes sociaux. Les papy-boomers n'ont-ils pas, en définitive, trop bien préparé leur retraite ? Et ne faudrait-il pas, tout simplement, remplacer dans le vocabulaire de l'analyse économique le triplet crise-déflation-austérité par papy-boom ? Espérons qu'il soit possible de poser la question de la solidarité intergénérationnelle des ainés envers les jeunes générations. Mais cela n'est pas certain.

${ }^{25}$ Le nombre de décès par an était de 540000 en 2014 ; il devrait augmenter pour atteindre 640000 en 2030. 


\section{Bibliographie}

Aalbers M, Christophers B (2014). Centring Housing in Political Economy. Housing, Theory \& Society 31(4) : 373-394

ABEL, ANDREW B., 2003. "The effects of a baby boom on stock prices and capital accumulation in the presence of social security". Econometrica

Allais, MAURICE, 1947. Economie et intérêt. Imprimerie Nationale, Paris.

Ando, Modigliani. (1963). "The "life-cycle" hypothesis of saving: aggregate implications and tests". The American economic review. 53(1) : 55-84.

ANG, MADDALONI. 2005. "Do demographic changes affect risk premiums? Evidence from International Data". Journal of Business

BeAubrun-Diant, Maury. (2015). "Quels sont les effets du prêt à taux zéro sur les prix du foncier?". EDHEC Position paper.

Bonnet O., Bono P.-H., Chapelle G., WASMER E. (2014). "Le capital-logement contribue-t-il aux inégalités? Retour sur Le capital au XXIe siècle de Thomas Piketty”. LIEPP Working Paper, 25.

BONO et TRANNOY (2012), «Evaluation de l'impact du dispositif Scellier sur les prix fonciers », Document de travail, Université Aix-Marseille.

CAMPBELL, 2007. "How do house prices affect consumption? Evidence from micro data". Journal of Monetary Economics

D’AlBis H., Moosa D., 2015, "Generational Economics and the National Transfer Accounts", Journal of Demographic Economics, 81, pp. 409-441.

DiAmond, Peter, 1965. National debt in a neoclassical growth model. American Economic Review 41 (1965), 1126-1150.

El KAROUI, 2013. “La lute des âges”. Flammarion

ENGElHARDT, GARY, POTERBA, JAMES M.. 1991. "Demographics and house prices: the Canadian evidence”. Regional Science and Urban Economics.

ERMISCH. (1996). "The demand for housing in Britain and population ageing: microeconometric evidence". Econometrica 63 : 383-404

FORTIN, LECLERC. 2002. "Déterminants du prix réel des logements au Canada". Actualité Economique, Société Canadienne de Science Economique

HALBERT L (2013). Les acteurs des marchés financiers font-ils la ville ? Vers un agenda de recherche. EspacesTemps.net, 9 juillet 2013

Halbert L, Henneberry J, Mouzakis F (2014). Finance, Business Property and Urban and Regional Development. Regional Studies 48(3) : 421-424

HARRIS, TZAVALIS (1999). "Inference for unit roots in dynamic panels where the time dimension is fixed". Journal of Econometrics 91(2) : 201-226.

HENDERSHOTT, PATRIC H.. 1991. “Are real house prices likely to decline by 47 percent? ”. Regional Science and Urban Economics. 
Holland. (1991). "The baby-boom and the housing market: Another look at the evidence". Regional Science and Urban Economics. 21(4) : 565-571

IM, PESERAN, SHIN. 2003. "Testing for unit root in heterogeneous panels". Journal of Econometrics.

JAMAL, QUAYES. 2004. “Demographic structure and stock prices”. Economics Letters

LANGUILLON-AUSSEL. 2015. "La renaissance urbaine dans une ville globale mature: vers un rétrécissement spatial de la rentabilité des investissements immobiliers à Tokyo”. Document de travail, Université Lyon 2.

Le BAyon, S., LeVASSEur, S., MADEC, P. (2013). “Achat de la résidence principale. Le profil des ménages français dans les années 2000”. Revue de l'OFCE / Débats et politiques. 128. pp. 451481.

LeE, Schmidt-Dengler, Felderer, Helmenstein. 2001. "Austrian Demography and Housing Demand: Is There a Connection?” Empirica. 28(3):259-276, 2001.

LEVIN, LIN, CHU. 2002. "Unit root tests in panel data: asymptotic and finite sample properties". Journal of Econometrics. 108 : 1-24.

MAnKIW, N.G., WeIL, D.N., 1989. "The baby boom, the baby bust and the housing market". Regional Science and Urban Economics.

MAREKWICA. MAURER, SEBASTIAN. 2011, “Asset meltdown-Fact or fiction”. Journal of Real Estate Portfolio Management

MAZUY M., BARBIERI M., BRETON D., D’Albis H., 2015, « L'évolution démographique récente de la France et ses tendances depuis 70 ans », Population, 70(3), pp. 417-486.

NAKAmura, K. and Y. SAITA (2007), "Land Prices and Fundamentals" Bank of Japan, Working Paper Series 07-E-8.

Neuteboom, P., Brounen, D. "Demography and housing demand - dutch cohort evidence". Erasmus University Working Paper, 2007.

NishimurA, K. G. (2011), "Population Ageing, Macroeconomic Crisis and Policy Challenges", presented at the Panel on "The General Theory and the Policy Reponses to Macroeconomic Crisis" at the 75th Anniversary Conference of Keynes' General Theory, University of Cambridge, June 19-21, 2011.

OHTAKE, SHINTANI. 1996. "The effect of demographics on the Japanese housing market". Regional Science and Urban Economics.

PASQUale, WheATON. (1994). "Housing market dynamics and the future of housing prices". Journal of Urban Economics. 35(1) : 1-27.

Pedroni P. (2004). "Panel co-integration: Asymptotic and finite sample properties of pooled time series tests with an application to the PPP hypothesis." Econometric Theory 20, pp. 597-625.

PIKeTTy, T. (2013). "Le capital au XXI siècle”. Editions du Seuil.

POTERBA, JAMES M.. 2001. “Demographic structure and asset returns”. The Review of Economics and Statistics.

POTERBA, JAMES M.. 2004. "Impact of population ageing on financial markets in developed countries". Federal Reserve Bank of Kansas City Economic Review.

Saita, Yumi, Shimizu, Chiniro, Watanabe, Tsutomu. 2013. "Aging and real estate prices: evidence from Japanese and US regional data”. Working paper. 
SAMUELSON, Paul A., 1958. "An exact consumption-loan model of interest with or without the social contrivance of money". Journal of Political Economy 66 (December), 467-482.

SHAN. 2012. "Reversing the trend: the recent expansion of the reverse mortgage market" Real Estate Economics 39(4) : 743-768

Shimizu, C and T. Watanabe (2010), "Housing Bubble in Japan and the United States", Public Policy Review Vol. 6, No. 3, 431-472.

TAKÁTS, Elöd. 2012, “Aging and housing prices”, Journal of housing economics.

YATES. 2011. “Cyclical versus Structural Sustainability of Homeownership: Is Counter-cyclical Intervention in Housing Markets Enough?” Housing studies 26 (7-8): 1059-1080 